\title{
Efficacy of toluidine blue staining in cervicovaginal cytology over conventional papanicolaou stain
}

\author{
Prakash V Patil ${ }^{1}$, Dhiraj B Nikumbh ${ }^{2, *}$ \\ ${ }^{1}$ Professor and Principal, ${ }^{2}$ Professor, ${ }^{1,2}$ Dept. of Pathology, JMF’s ACPM Medical College, Dhule, Maharashtra, India
}

*Corresponding Author:

Email: drdhirajnikumbh@ rediffmail.com

\begin{abstract}
Introduction: Toluidine blue staining (TBS) is a practical, rapid, inexpensive and effective adjunct diagnostic tool. TBS method has been extensively used as a vital stain with metachromatic property for mucosal lesions and exfoliative cytology.

Aim and Objectives: To see the efficacy of aqueous Toluidine Blue (TB) stained smears in comparison to conventional smears stained with Papanicolaou (Pap) stain of cervicovaginal smears and to reduce the reporting time of smears and also cutting down on the cost.

Materials and Methods: This is a prospective cross sectional study on 240 Cervicovaginal smears received in the Dept. of Pathology, ACPM Medical College College, Dhule over a period of 4 months from September to December 2016. All the satisfactory smears as per Bethesda system of reporting were included in the study. The unsatisfactory smears were excluded from the study. Multiple smears from the symptomatic patients were advised. The conventional Pap stain and Aqueous TBS (1\%) was performed on the smears received. Cytomorphology of the smears were studied and compared in reference to staining, timing and efficiency of stains.

Results: The efficacy of TBS is equally good as conventional Pap staining. The timing is curtailed from average 30 minutes in Pap staining to 3 minutes in TB staining method. The cost per test was also decreased substantially in TB Staining method. The nuclear staining property by TBS is equally good as in Pap staining most of the times, for rapid diagnosis of lesion.

Conclusion: The staining properties of TB Stain are similar and equally good as Pap staining method with reduced turnaround time and are cost effective. Hence TB staining method is beneficial for mass screening of cervicovaginal smears in the camps in Indian scenario.
\end{abstract}

Keywords: Toluidine blue, Pap smears, Cervicovaginal smears, Staining.

\section{Introduction}

Toluidine blue (TB) stain is a basic metachromatic dye with high affinity for acidic components like nucleic acids and therefore binds to nuclear material of tissues with a high DNA and RNA content. ${ }^{1}$ TBS is partially soluble in both water and alcohol and is a member of the thiazine group. ${ }^{2}$ In 1856, TBS was discovered by William Henry Perkin, after it was primarily used by dye industry. ${ }^{3}$ TBS has been extensively used by various researchers as a vital stain for oral mucosal lesion and aspiration cytology reporting in recent years. ${ }^{3-5}$ The literature shows, TBS is a practical, rapid, inexpensive and effective adjunct diagnostic tool. ${ }^{\text {4- } 6}$

Toluidine blue is supravital stain that accentuates good cytological and nuclear details and enables the three dimensional view of cells in wet mount film. It is easily available, very cheap, cost effective and used for quick reporting. ${ }^{4,6}$ TBS is based on principle of metachromasia. Various applications of TBS are demonstration of connective tissue mucins, mast cell granules, amyloid, H.Pylori, endocrine cell granules, frozen section staining, etc. ${ }^{3}$

To the best of our knowledge, most of the studies of Toluidine blue were done on FNA cytology and oral mucosal lesions with no study published in cervicovaginal cytology. Hence we undertook this study on Cervicovaginal cytology, to see the efficiency of Toluidine blue staining over conventional Pap staining method. The reporting timing also reduced using the Toluidine blue staining. It is cost effective, rapid staining and simple, reliable method for mass screening in camps.

\section{Materials and Methods}

This is a prospective, cross sectional study conducted on cervicovaginal smears received in the department of Pathology, ACPM Medical College, Dhule over a period of 4 months from September to December 2016. A total of 240 consecutive symptomatic patients refereed to the gynecology department for Pap smears with satisfactory smears as per The Bethesda system of reporting (2014) were included in the study. The unsatisfactory smears were excluded from the present study.

Multiple smears (preferably two/three) were advised to be collect in symptomatic patients in the gynecology department of our hospital. The smears were sent in Coplin jar containing iso propyl alcohol. Smears are fixed for minimum of 10 minutes

The conventional Pap staining and aqueous $1 \%$ Toluidine blue staining on the slides received was done. For Toluidine Blue staining procedure, aqueous $1 \%$ TBS was prepared by mixing 1 gram of Toluidine blue powder in $100 \mathrm{ml}$ of distilled water.

After fixation, the slides are dipped in $50 \%$ alcohol for $1 \mathrm{~min}$, then water washed. Smears and then stained with $1 \%$ aqueous TBS for 45 to 60 seconds. Slides are water 
washed, air dried, clear with xylene and mount in DPX. ${ }^{6,7}$

The aqueous $1 \%$ Toluidine blue stained slides and Pap stained smears were compared with respect to staining properties, efficacy and timing of reporting of smears.

\section{Results}

In the present study, total of 240 cases were subjected to conventional Pap staining and aqueous $1 \%$ TBS over a period of 4 months. The mean age was 33 years in study range from 19 years to 66 years. The cervicovaginal smears were reported by the Bethesda system of reporting $(2014)^{8}$ as shown in Table 1. Negative for intraepithelial lesion /malignancy (NILM) was reported in $94.7 \%$ cases. ASC-US was in $21 \%$, LSIL was in $1.2 \%$ and HSIL was observed in $0.8 \%$ in the present study. 2 cases $(0.8 \%)$ of ASC-H and one case of Squamous cell carcinoma $(0.4 \%)$ were reported.
In NILM category, non-specific inflammation $(67.9 \%)$ was most common finding as shown in Table 2. Bacterial vaginosis was seen in $21.2 \%$, Candida albicans was in $4.4 \%$, Trichimonas vaginalis infection was in $1.3 \%$ and atrophic vaginitis seen in $5.2 \%$ in our study.

The staining properties, diagnostic efficacy and reporting time of conventional Pap staining and aqueous $1 \%$ TBS was studied. The staining properties, especially nuclear features in specific lesions were better in TBS over conventional Pap stain.

The overall reporting time for aqueous $1 \%$ TBS was 5 minutes after fixation, whereas the conventional Pap stain requires 30 minutes.

With respect to staining properties and reporting time, TBS is advantageous over Pap staining method. The turnaround time (TAT) will be reduced in TBS as compared to conventional Pap staining in mass screening during camps. Reports are immediately given to suggest the further line of treatment.

Table 1: Cervicovaginal cytology interpretation by the Bethesda system

\begin{tabular}{|l|c|c|c|}
\hline S. No. & Cytological interpretation & No. of cases & Percentage (\%) \\
\hline 1 & $\begin{array}{c}\text { Negative for intraepithelial } \\
\text { lesion(NILM) }\end{array}$ & 227 & 94.7 \\
\hline 2 & ASC-US & 05 & 2.1 \\
\hline 3 & LSIL & 03 & 1.2 \\
\hline 4 & HSIL & 02 & 0.8 \\
\hline 5 & ASC-H & 02 & 0.8 \\
\hline 6 & Squamous cell carcinoma & 01 & 0.4 \\
\hline \multicolumn{2}{|c|}{ Total } & $\mathbf{2 4 0}$ & $\mathbf{1 0 0}$ \\
\hline
\end{tabular}

Table 2: Categorization of negative for intraepithelial lesion (NILM)

\begin{tabular}{|l|c|c|c|}
\hline S.No. & Cytological diagnosis & No. of cases & Percentage (\%) \\
\hline 1 & Non-specific inflammation & 154 & 67.9 \\
\hline 2 & Bacterial vaginosis & 48 & 21.2 \\
\hline 3 & Candida albicans & 10 & 4.4 \\
\hline 4 & Trichomanas vaginalis & 03 & 1.3 \\
\hline 5 & Atrophic vaginitis & 12 & 5.2 \\
\hline & Total & $\mathbf{2 2 7}$ & $\mathbf{1 0 0}$ \\
\hline
\end{tabular}

\section{Discussion}

In cervicovaginal cytology, we evaluated the utility of Toluidine blue stain smears in comparison over conventional Pap stained smear to increase the diagnostic efficacy and decrease the reporting time. The present study was designed to evaluate the staining properties helpful for mass screening in short span of time for specific inflammation as well as SIL lesions.

The biggest advantage of the TBS staining was that it can be used at procedure site as well as in screening camps in view of its simple procedure, requires less staining time and reporting turnaround time as compared to conventional Pap staining. TBS required hardly 5 minutes and reports issued to the patient within 10 minutes as compared to Pap staining method which required 30 minutes for staining only. The reports are dispatched to the patients after several hours or next day depending on availability of resources in Pap staining. Secondly, the Pap staining method is rarely useful for onsite procedure in view of its lengthy procedure and requires skilled technician. Hence TBS study can be used for mass screening due to its additional edge over Pap staining method vis a vis effortless staining and reduced turnaround time.

In 2015, Saba k et al ${ }^{4}$ used the TBS staining in fine needle aspiration cytology (FNAC) and concluded that TBS study of FNAC improves the diagnostic accuracy by minimizing the smearing and drying artifact, loss of cell sample during fixation and staining also increases diagnostic accuracy. In 2012, Ammanagi AS et $\mathrm{al}^{5}$ showed that on site toluidine blue staining and screening improved the efficiency of FNAC reporting. 
The various researchers like Miller RL et $\mathrm{al}^{9}$, Onofre MA et al, ${ }^{10}$ Mashberg $\mathrm{A}^{11}$ and Hedge $\mathrm{CM}$ et al ${ }^{12}$ proved in their respective articles TBS as diagnostic adjunct in detection of asymptomatic oral squamous cell carcinoma and its utility in early detection of oral malignancies. Furthermore Warnakulasuriya KA el al ${ }^{13}$ discussed the role of toluidine blue mouth rinse in oral cancers.

We studied 240 cases of cervicovaginal cytology smears and compared the two stains, TBS with conventional Pap stain. In majority of cases of NILM (94.7\%), we observed that inflammatory exudates were predominantly noted in TBS as compared to Pap stained smears. It was prominent and even microscopic details like phagocytosis in polymorphs with inflammatory background was better appreciated in TBS. Regarding specific inflammation, in Candida albicans, the budding yeast was predominantly seen in TBS and actually they are confirmed in TBS stain as they may confuse with RBCs in conventional Pap stained smear (Fig. $1 \mathrm{a}, \mathrm{b}$ ). As in Bacterial vaginosis (21.2\%) the characteristic features of 'Clue cell' was strikingly noted in TBS (Fig. 1 c, d). Trichomas vaginalis organisms with characteristic morphology pear shaped with blue bob appearance highlighted in TBS (Fig. 1e). HSV showed characteristic ground glass nuclei and multinucleation (Fig. $1 \mathrm{f}$ ) favoring more of LSIL category.
In SIL category, we observed the biggest advantage of TBS in LSIL (3 cases). The characteristic Koilocytic change -perinuclear (clearing) vacuolization with nuclear features were prominently seen in TBS (Fig 2 c, d). In HSIL (2 cases), the nuclear and cytoplasmic properties of dyskaryosis were highlighted in TBS, whereas it was faint stain due to overcrowding of nuclei (Fig. 2 e, f). Same was true for ASCUS (5 cases), nuclear and cytoplasmic characteristics was better in TBS (Fig. 2 a, b). We encountered a single case of squamous cell carcinoma (SCC) and the characteristic features like strap cell, tadpole cell (Fig. 3 a, c) with all neoplastic features of SCC like pleomorphic hyperchromatic nuclei, irregular nuclear chromatin, prominent nucleoli were more highlighted in TBS over pap stain (Fig. 3 b, d ). We found $5.2 \%$ cases of atrophic vaginitis and in these the features of parabasal cells, mild anisonucleosis were more clearly seen in TBS to avoid the over diagnosis in Pap stained smear (Fig. 3 e, f).

Toluidine blue stain is useful to overcome some limitations of cervicovaginal cytology with respect to technical aspect as published in Pap society and SAJC by Nikumbh DB et al. ${ }^{14,15}$ It may be of great help for mass screening of cervical cancers where cervical cancer is "preventable but not prevented" yet and it is the reality in India as quoted by Miniello G and Saraiya U. ${ }^{16}$

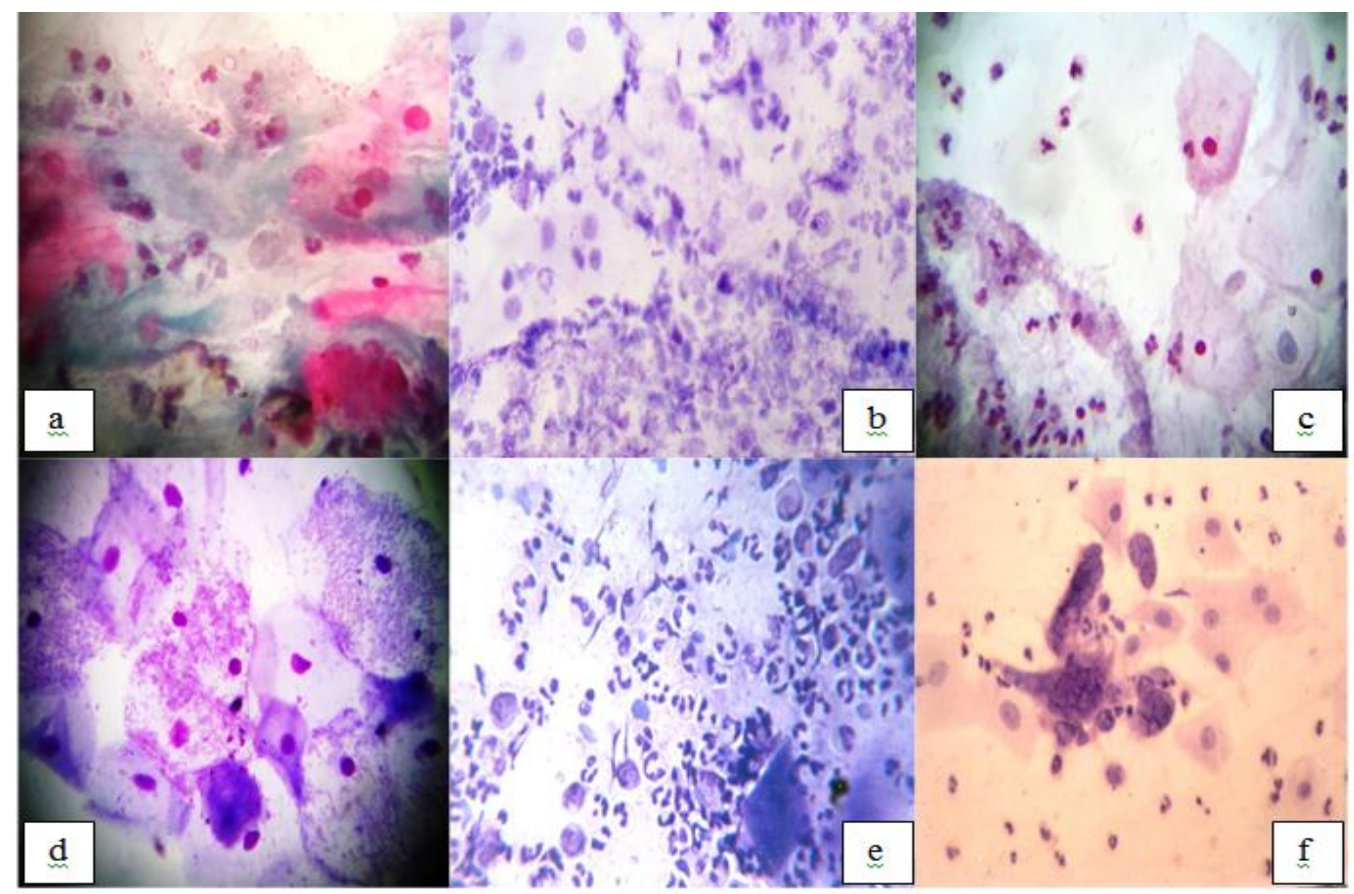

Fig 1: Photomicrograph showing: a) Budding yeast of Candida albicans not highlighted (Pap, x400); b) Characteristic budding yeast prominently seen (TBS, $x 400)$; c) Bacterial vaginosis inflammation (Pap, $x 400)$; d) Clue cell clearly seen ((TBS, x400); e) Trichomonas vaginalis infection (TBS, x400); f) HSV infection with ground glass nuclei and multinucleation (TBS, $x 400)$ 


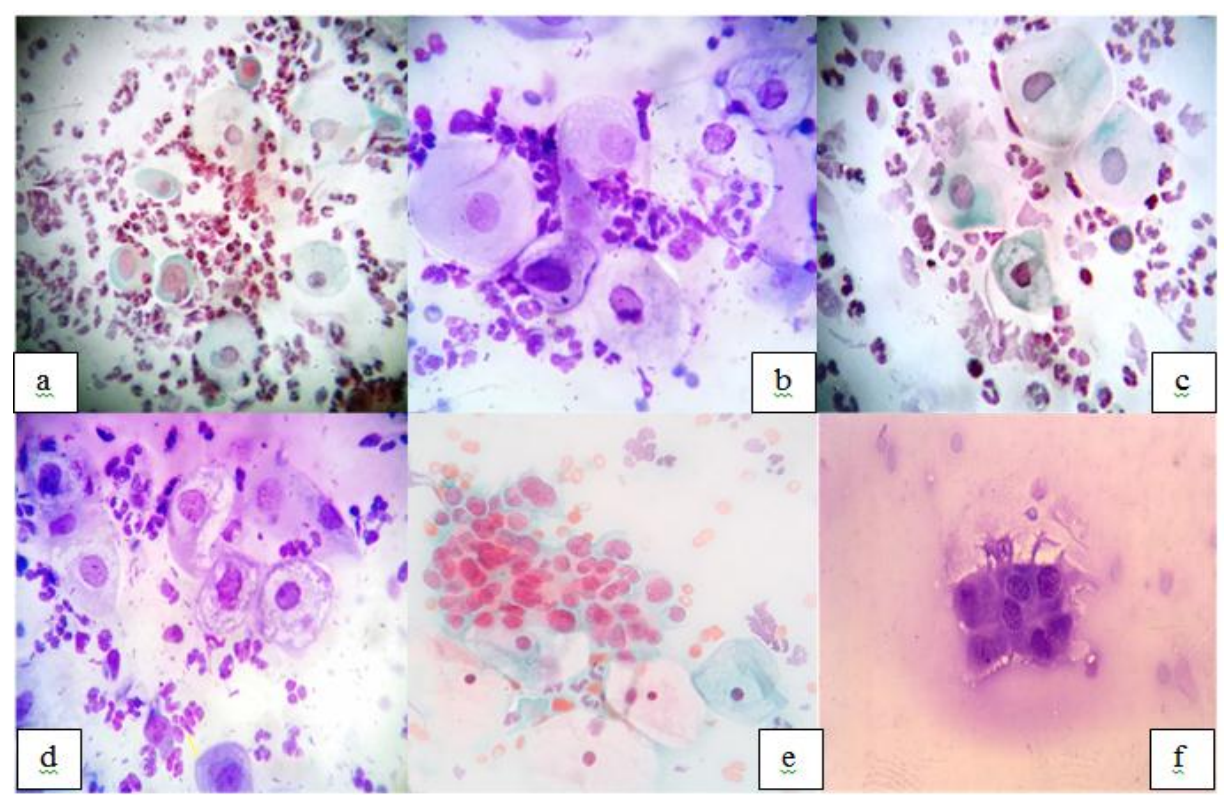

Fig. 2: Photomicrograph showing; a) ASCUS with nucleomegaly (Pap, x400); b) Characteristics of ASCUS clearly seen (TBS, x400); c) LSIL with koilocytic change (Pap, x400); d) Perinuclear clearing with nuclear features highlighted (TBS, x400); e) HSIL with crowded nuclei (Pap, x400); f) Nuclear changes of HSIL clearly seen (TBS, $\mathbf{x 4 0 0 )}$ )

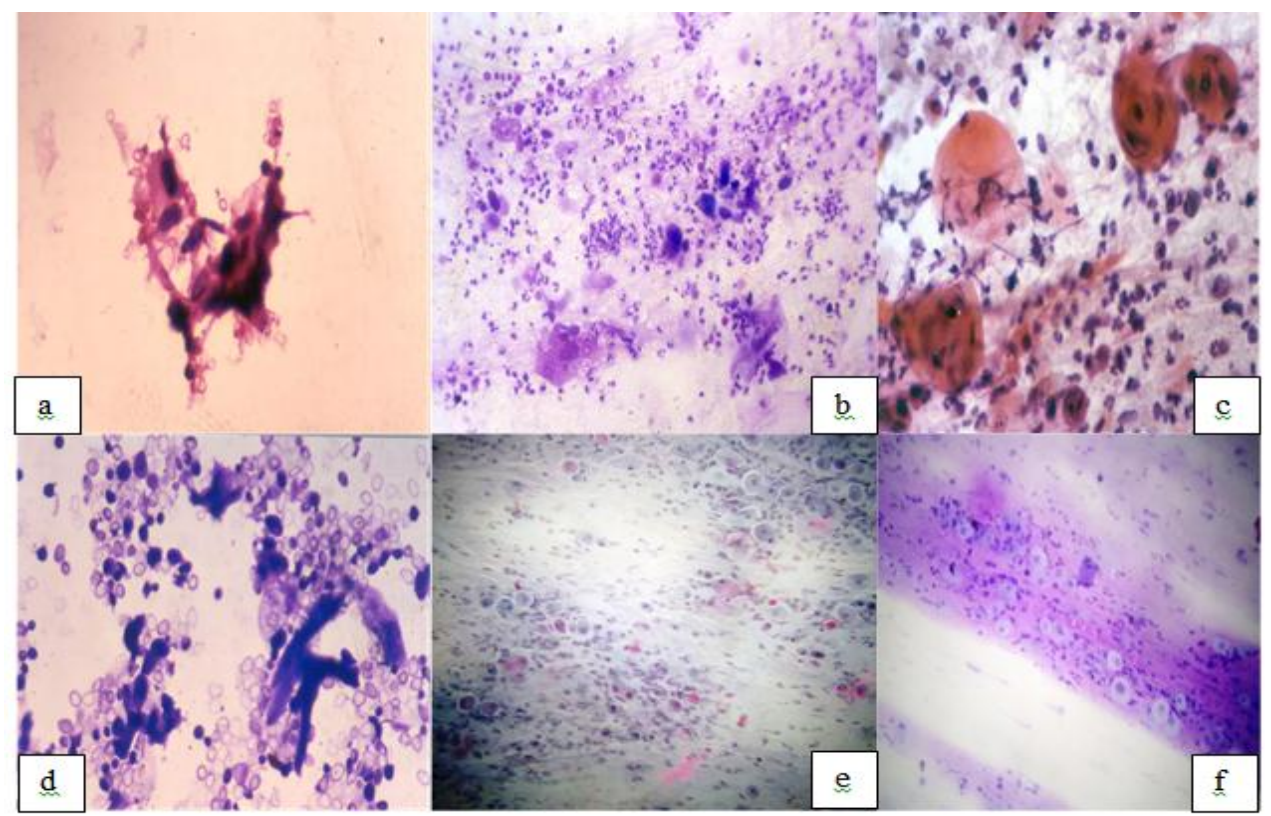

Fig. 3: Photomicrograph showing: a,c) SCC showing dyskaryosis (Pap, x400); b,d) SCC with tadpole, strap cells highlighted (TBS, x400); e) Atrophic smear with parabasal cells (Pap, x100); f) Parabasal cells prominently seen with inflammation (TBS, x100)

\section{Conclusion}

Taking into consideration all of the above findings and diagnostic properties of TBS, we conclude that the TBS is better for cervicovaginal cytology.TBS is preferred over Pap staining with respect to staining properties of nucleus as well as cytoplasm and more so in the NILM category. TBS is advantageous due to quick reporting in routine practice and especially in mass Pap smear screening camps.
Acknowledge: We acknowledge Dr Vijay Karnik and Dr Kunda Karnik, Consultant Pathologist, Pune for their innovative and technical idea.

\section{Conflict of Interest: Nil}

Source of Funding: Nil 


\section{References}

1. Epstein JB, Oakley C, Millner A, Emerton S, vander Meij $\mathrm{E}, \mathrm{Le} \mathrm{N}$. The utility of toluidine blue application as a diagnostic aid in patients previously treated for upper oropharyngeal carcinoma. Oral Surg Oral Med Oral Pathol Oral Radiol Endod. 1997;83:537-47.

2. Gandalfo S, Pentenero M, Broccoletti R, Pagano M, Carrozzo M, Scully C. Toluidine blue uptake in potentially malignant lesions in vivo: Clinical and histological assessment. Oral Oncol. 2006;42:89-95.

3. Sridharan G and Shankar AA. Toluidine blue: A review of its chemistry and clinical utility. J Oral Maxillofac Pathol. 2012 May-Aug;16(2):251-255.

4. Saba K, Niazi S, Bukhari MH, and Imam SF. Use of supravital toluidine blue staining to improve the efficiency of fine-needle aspiration cytology reporting in comparison to papanicolaou stain. Pak J Med Sci. 2015 Sep-Oct;31(5):1146-1151.

5. Ammanagi AS, Dombale VD, Patil SS. On -site toluidine blue staining and screening improves efficiency of fine needle aspiration cytology reporting. Acta Cytol. 2012;56(4):347-51.

6. Naylor B. Pleural, Peritoneal and Pericardial Effusions. in: Bibbo Marluce, Wilburg David (Editors), Comprehensive Cytopathology. $3^{\text {rd }}$ Ed. Saunders Elsevier,_Philadelphia. 2008:515-577.

7. Alan Stevans. Standard toluidine blue method. Amyloid. in: Bancroft JD, Stevans A (editors). Theory and practice of histological technique. $3^{\text {rd }}$ ed. Churchill Livingstone, New York. 1990:167.

8. Nayar R., Wilbur D.C. The Pap Test and Bethesda 2014. Acta Cytologica 2015;59:121-132.
9. Miller RL, Simms BW, Gould AR. Toluidine blue staining for detection of oral premalignant lesions and carcinomas. J Oral Pathol Med. 1988;17:73-8.

10. Onofre MA, Sposto MR, Navarro CM. Reliability of toluidine blue application in the detection of oral epithelial dysplasia and in situ and invasive squamous cell carcinomas. Oral Surg Oral Med Oral Pathol Oral Radiol Endod. 2001;91:535-40.

11. Mashberg A. Reevaluation of toluidine blue application as A diagnostic adjunct in the detection of asymptomatic oral squamous cell carcinoma: a continuing prospective study of oral cancer III. Cancer. 1980;46:758-63.

12. Hegde CM, Kamath PN, Sreedharan S, Dannana NK, Raju RM. Supravital staining: It's role in detecting early malignancies. Indian J Otolaryngol Head Neck Surg. 2006;58:31-4.

13. Warnakulasuriya KA, Johnson NW. Sensitivity and specificity of OraScanÒ toluidine blue mouthrinse in the detection of oral cancer and precancer. J Oral Pathol Med. 1996;25:97-103.

14. Nikumbh DB, Kesavaperumal V and Dewar R.View point.Cytology based screening in India. Focus Newsletter. Papanicolaou Society of Cytopathology. Pap society.Org 2012;16(2):14-16.

15. Nikumbh DB, Nikumbh RD, and Kanthikar SN. Limitations of cytological cervical cancer screening (Papanicolaou test) regarding technical and cultural aspect in rural India. South Asian J Cancer. $2016 \mathrm{Apr}-$ Jun;5(2):79.

16. Miniello G, Saraiya U. 1st ed. New Dehli: CBC Pub; 1999. Historical survey and basic cytology. Colour Atlas of Cytology and Colposcopy; pp. 1-22. 